\title{
Potential high-frequency off-target mutagenesis induced by CRISPR/ Cas9 in Arabidopsis and its prevention
}

\author{
Qiang Zhang ${ }^{1} \cdot$ Hui-Li Xing ${ }^{1} \cdot$ Zhi-Ping Wang $^{1} \cdot$ Hai-Yan Zhang ${ }^{1} \cdot$ Fang Yang $^{1} \cdot$ Xue-Chen Wang ${ }^{1} \cdot$ Qi-Jun Chen ${ }^{1}(\mathbb{D}$
}

Received: 23 December 2017 / Accepted: 6 February 2018 / Published online: 23 February 2018

(c) The Author(s) 2018. This article is an open access publication

\begin{abstract}
Key message We present novel observations of high-specificity SpCas9 variants, sgRNA expression strategies based on mutant sgRNA scaffold and tRNA processing system, and CRISPR/Cas9-mediated T-DNA integrations.

Abstract Specificity of CRISPR/Cas9 tools has been a major concern along with the reports of their successful applications. We report unexpected observations of high frequency off-target mutagenesis induced by CRISPR/Cas9 in T1 Arabidopsis mutants although the sgRNA was predicted to have a high specificity score. We also present evidence that the off-target effects were further exacerbated in the T2 progeny. To prevent the off-target effects, we tested and optimized two strategies in Arabidopsis, including introduction of a mCherry cassette for a simple and reliable isolation of Cas9-free mutants and the use of highly specific mutant SpCas9 variants. Optimization of the mCherry vectors and subsequent validation found that fusion of tRNA with the mutant rather than the original sgRNA scaffold significantly improves editing efficiency. We then examined the editing efficiency of eight high-specificity SpCas9 variants in combination with the improved tRNA-sgRNA fusion strategy. Our results suggest that highly specific SpCas9 variants require a higher level of expression than their wildtype counterpart to maintain high editing efficiency. Additionally, we demonstrate that T-DNA can be inserted into the cleavage sites of CRISPR/Cas9 targets with high frequency. Altogether, our results suggest that in plants, continuous attention should be paid to off-target effects induced by CRISPR/Cas9 in current and subsequent generations, and that the tools optimized in this report will be useful in improving genome editing efficiency and specificity in plants and other organisms.
\end{abstract}

Keywords Genome editing · CRISPR/Cas9 · Off-target effects · Editing specificity · Editing efficiency

\section{Introduction}

CRISPR-Cas9 from microbial adaptive immune systems is a powerful tool for genome editing in a variety of organisms (Carroll and Charo 2015; Cong et al. 2013; Jinek et al. 2012; Mali et al. 2013). In plants, various CRISPR/Cas9based applications to basic research and crop breeding have been launched (Yin et al. 2017). For example, decades of

Qiang Zhang and Hui-Li Xing contributed equally to this work.

Electronic supplementary material The online version of this article (https://doi.org/10.1007/s11103-018-0709-x) contains supplementary material, which is available to authorized users.

Qi-Jun Chen

qjchen@cau.edu.cn

1 State Key Laboratory of Plant Physiology and Biochemistry, College of Biological Sciences, China Agricultural

University, Beijing 100193, China debate on the function of $A B P I$ have been resolved through CRISPR/Cas9 (Gao et al. 2015). Further analysis of the T-DNA insertion mutant of $A B P 1$ (abpl-1) found that the T-DNA insertion event also caused unwanted mutation in the adjacent $B S M$ gene, thereby causing the mutant phenotype of embryonic lethality (Dai et al. 2015). Since the CRISPR/Cas9 system corrected the mistake made by the T-DNA insertion, it seemed that the CRISPR/Cas9 system was perfect. Nevertheless, the CRISPR/Cas9 system can also potentially make similar mistakes, i.e., causing undesirable mutations or off-target effects (Carroll 2013). Thus, the case of $A B P 1$ not only demonstrates the huge potency of CRISPR/Cas9 tools but also provides a lesson from off-target mutagenesis to users of the tools. In addition, assessing the specificity of CRISPR/Cas9 to avoid the risk of off-target mutations, e.g., unanticipated downstream effects, is also an important regulatory concern for agricultural applications (Wolt et al. 2016). 
High-frequency off-target mutagenesis induced by CRISPR/Cas9 has been reported in human cells (Fu et al. 2013, Hsu et al. 2013; O'Geen et al. 2015; Tsai and Joung 2016; Tycko et al. 2016; Wu et al. 2014). However, it seems that off-target mutations in plants are rare and only a few cases representing low-frequency off-target mutations have been reported (Wolt et al. 2016). Whole genome sequencing (WGS) of Arabidopsis and rice mutants has shown that the CRISPR/Cas9 system is highly specific in plants (Feng et al. 2014; Zhang et al. 2014). Deep sequencing of a total of 178 off-target sites further demonstrated that multiplex targeting in Arabidopsis is highly specific to on-target sites with no detectable off-target events (Peterson et al. 2016).

Seemingly contrary to these reports, the present study reports unexpected observations of high-frequency off-target mutations induced by a sgRNA with a specificity score as high as 94 relative to the range of 0-100 (Haeussler et al. 2016, Hsu et al. 2013). Furthermore, we also describe the optimized strategies to avoid off-target mutations. Finally, we discussed the reasons for the inconsistency between this report and the previous reports.

\section{Results}

\section{Unexpected observations of high-frequency off-target mutagenesis induced by CRISPR/Cas9}

We previously reported the generation of 15 mutant T1 lines harboring EC1fp:Cas9 and two sgRNAs targeting the three genes: TRY, CPC , and ETC2 (Wang et al. 2015). When we analyzed mutations in the three genes in these 15 mutant plants, we were able to obtain the ETC2 fragment in all the 15 lines, but not able to obtain the TRY fragment from one line (\#6) and the $C P C$ fragment from 7 lines (\#2, 4, 6, 8, 11, 13 , and 14). After the repeated failures in the PCR amplifications, we suspected that off-target mutations might be the reason for the failures. Next, we re-conducted PCR amplifications using a modified primer pair (-FP/-RP, Fig. 1) spanning the off-target and on-target sites instead of the original primer pair (-FP0/-RP, Fig. 1). As a result, we successfully obtained the PCR fragments from all the lines except for the $C P C$ gene in line \#4, and found that the $C P C$ fragments in some lines exhibited complex patterns (Fig. 1). Sequencing analysis further verified that the difficulties or failures in PCR amplification as well as the complexity of the PCR products were due to off-target mutations (Table S1). Thus, we unexpectedly found high frequency (87\%) off-target mutations in the $C P C$ gene during analysis of the mutations in the three target genes in the 15 lines. The specificity score based on the in silico prediction algorithm was 94 relative to the range of 0-100 (Haeussler et al. 2016, Hsu et al. 2013), suggesting that some sgRNAs predicted to have a high specificity score may still have possibility to induce highfrequency off-target mutations. The observed differences in mutation frequencies in the three potential off-target sites, which all harbor three mismatches to the sgRNA, could be explained by previously described rules (Tsai and Joung 2016; Tycko et al. 2016; Wu et al. 2014). The insurmountable failures in PCR amplification of the $C P C$ fragment from line \#4 can be attributed to T-DNA insertions, which will be described later in this report.

\section{Evidence of aggravated off-target effects in T2 progeny}

To analyze off-target mutations in T2 plants and to simplify the analysis, we first generated two new CRISPR/Cas9 vectors, each harboring only one-sgRNA cassette. Analysis of off-target mutations indicated that the frequency of off-target mutations in the newly generated $\mathrm{T} 1$ plants was not as high as that in the 15 lines harboring two sgRNA cassettes, which suggests that the off-target mutations were enhanced by adjacent on-target mutations. Nevertheless, the efficiencies of the off-target mutations in the $C P C$ gene of the newly generated transgenic plants were still higher than $10 \%$ (Fig. 2). In practice, upon detection of unwanted off-target mutations, researchers would always pay attention to those from intended mutants rather than normal plants. Therefore, we analyzed off-target mutations in T2 plants from six etc2 mutant lines (Fig. 2). The results indicated that off-target mutations in the $C P C$ gene of $\mathrm{T} 2$ plants were highly frequent and averaged higher than $60 \%$. Moreover, $3 \%$ of the T2 plants harbored off-target mutations in the two genes (CPC and TRY) (Fig. 2; Table S2). These results suggest that off-target effects were indeed aggravated in the $\mathrm{T} 2$ progeny.

\section{Optimization of the strategy for isolating Cas9-free mutants to overcome off-target mutations in the progeny}

To overcome the intensified effects of off-target mutations on the $\mathrm{T} 2$ progeny, we introduced a mCherry cassette into our CRISPR/Cas9 system for simple and reliable isolation of Cas9-free Arabidopsis mutants (Gao et al. 2016). Introduction of the mCherry vector harboring the original sgRNA scaffold (Fig. 3) lowered editing efficiency (2.4\%) relative to that observed in our previous study ( $>10 \%$ for the three target genes), suggesting that the mCherry cassette has a negative effect on the CRISPR/Cas9 system. We then made efforts to optimize the system. We used a mutant sgRNA scaffold (Dang et al. 2015), which increased editing efficiency from 2.4 to $3.8 \%$, whereas deletion of the $3 \times$ Flag of Cas 9 decreased efficiency to $1.7 \%$. Upon fusion of the mutant sgRNA scaffold to tRNA, a significant increase in editing efficiency was observed (Fig. 3). Thus, 
(A)

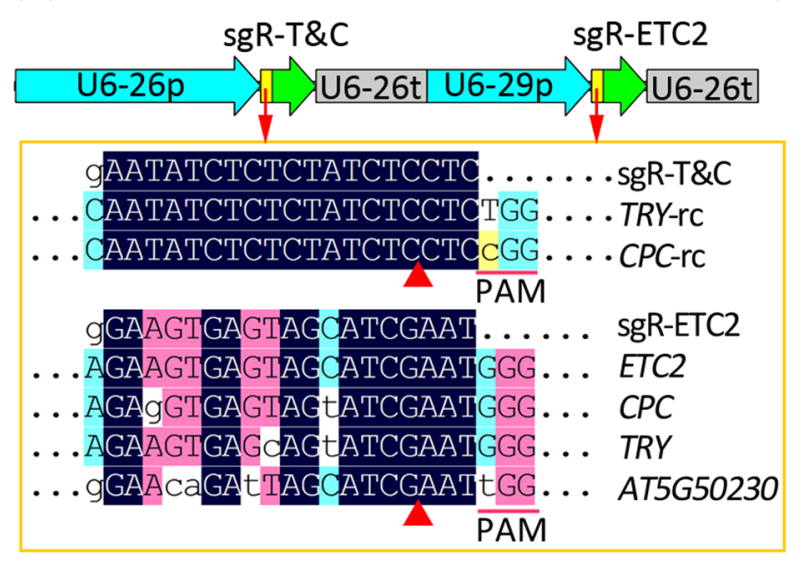

(C)

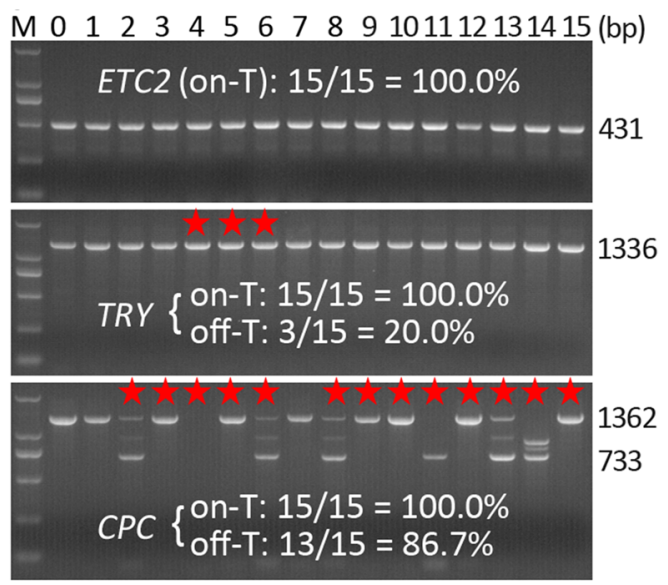

(B)

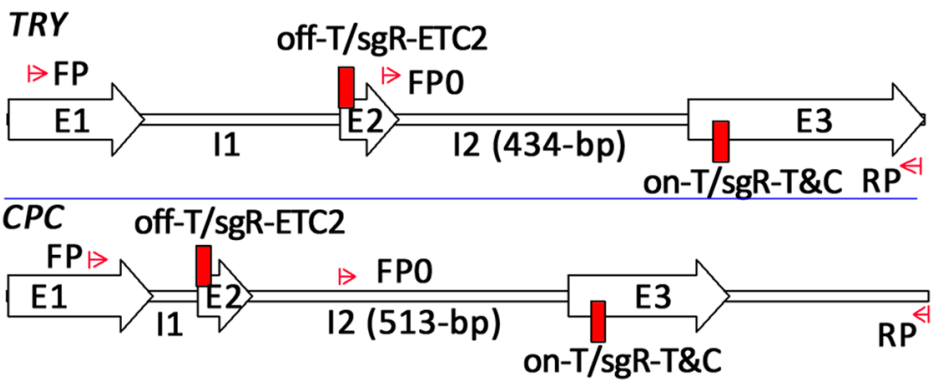

$E 1 / 2 / 3$, exon- $1 / 2 / 3$

$11 / 2$, intron- $1 / 2$

on/off-T, on/off-target

FP/RP, forward/reverse primer site

Fig. 1 CRISPR/Cas9-induced high-frequency off-target mutagenesis in Arabidopsis. a Two sgRNA expression cassettes and the alignment of the sgRNAs with their target and potential off-target genes in the 15 T1 likely try cpc etc 2 triple mutant lines reported previously. Only aligned regions of interest are displayed. -rc, reverse complement. Note: the $\operatorname{sgR}$-ETC2 has a specificity score of 94 relative to the range of $0-100$. b The sgRNA targeting ETC2 gene (sgR-ETC2) has poten- tial off-target sites in the two genes, $T R Y$ and $C P C$, which are targeted by the other sgRNA ( $\operatorname{sgR}-\mathrm{T} \& \mathrm{C})$. c PCR amplification with primers spanning on-target and off-target sites revealed the sgR-ETC2induced off-target mutations in the TRY and $C P C$ genes. The stars represent off-target mutations as determined by sequencing analysis. Note: failure in PCR amplification of the CPC gene in line \#4 was due to T-DNA insertions described later in this report

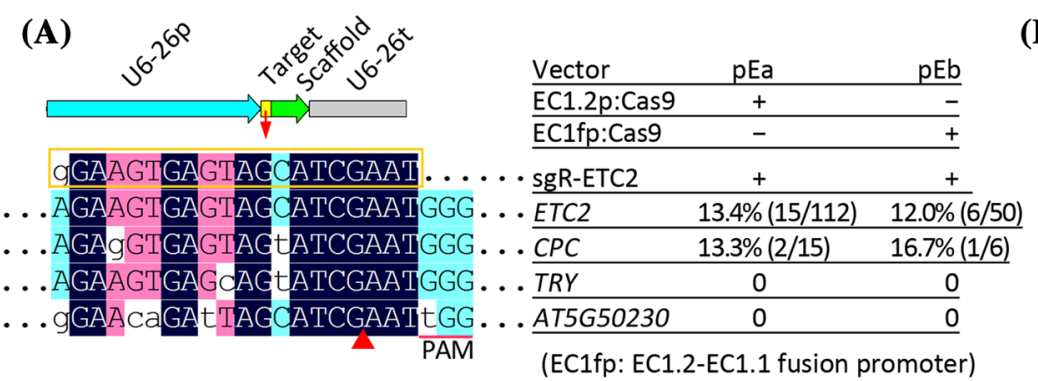

(EC1fp: EC1.2-EC1.1 fusion promoter)
(B)

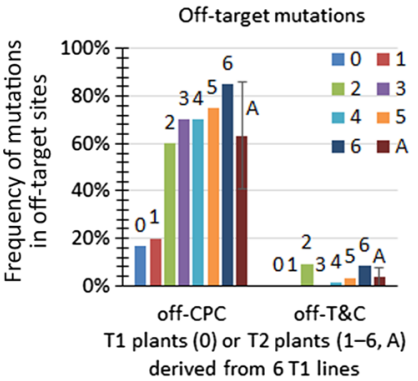

Fig. 2 Evidence of aggravated off-target effects in T2 progeny. a Ontarget and off-target mutation frequencies in T1 transgenic lines harboring a sgRNA expression cassette and one of the two types of Cas9 expression cassettes. The names of the two CRISPR/Cas9 binary vectors and their Cas9/sgRNA expression cassettes are indicated on the upper right. The aligned sequences of the sgRNA, target and off-target sites are displayed on the left panel. The mutation frequencies are indicated on the right of the on/off-target genes. The on-target mutation efficiency was calculated based on the ratio of number of mutants to total number of T1 plants. The off-target mutation frequency was calculated based on the ratio of number of the mutants harboring off- target mutations to total number of mutant plants. b Off-target effects were aggravated in T2 plants. $6 \mathrm{~T} 2$ populations from the $6 \mathrm{~T} 1 \mathrm{pEb}$ transgenic plants harboring ETC2 mutations were analyzed. For each $\mathrm{T} 2$ population, 20 randomly selected $\mathrm{T} 2$ plants were analyzed for offtarget mutations in the $C P C$ gene. The frequency of off-target mutations in two genes (CPC and TRY) was calculated based on the ratio of the number of mutant plants with clustered leaf trichomes to the total number of T2 plants examined. T1 line numbers $1-6$ correspond to original $\mathrm{T} 1$ line numbers $48,6,31,20,7$, and 43 , of which only \#20 harbors chimeric off-target mutations in the $C P C$ gene in $\mathrm{T} 1$ plants. $A$ average value for the six lines 


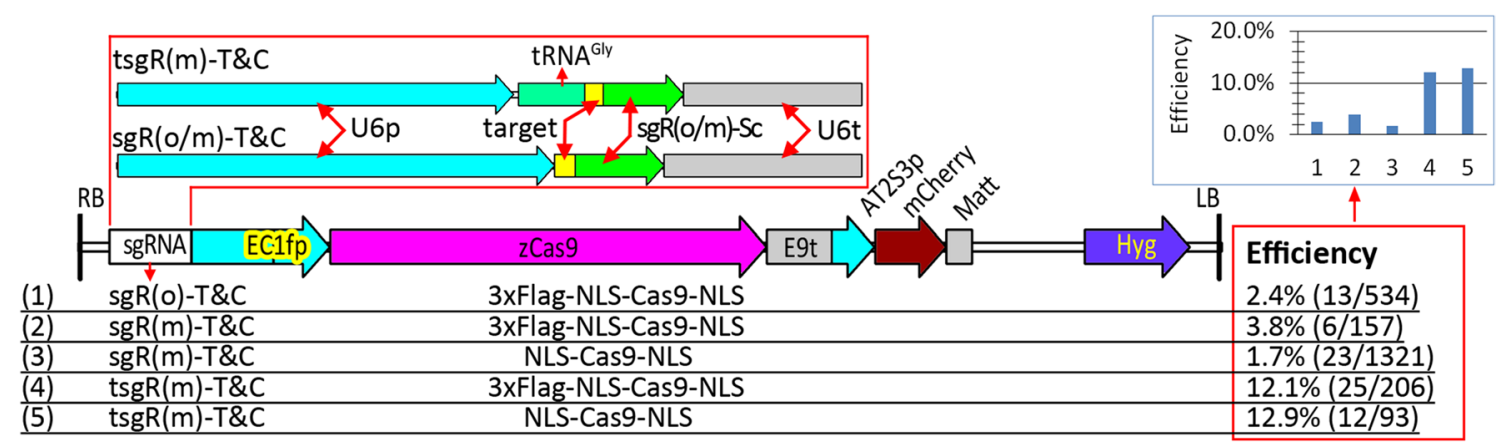

Fig. 3 The negative effect of the mCherry cassette on editing efficiency could be overcome by fusion of tRNA with mutant sgRNA scaffold. Five combinations of three types of sgRNA cassettes (targeting $T R Y$ and $C P C$ ) and two Cas9 variants with or without $3 \times$ Flag, their physical maps of T-DNA, and efficiencies of mutations in the

the combination of the two strategies based on the tRNA and mutant sgRNA scaffold can overcome the negative effects of the mCherry cassette on the CRISPR/Cas9 system.

\section{Fusion of tRNA with mutant sgRNA scaffold conferred a significantly higher editing efficiency than that using the original sgRNA scaffold}

The activities of the high-specificity SpCas9 variants have been shown to be highly sensitive to mismatches of sgRNAs to their targets; even when the first base of the sgRNAs were mismatched to their targets, editing efficiency of high-specificity SpCas9 significantly decreased (Kleinstiver et al. 2016; Slaymaker et al. 2016). Because the fused tRNA-sgRNAs were efficiently and precisely processed into sgRNAs with the desired 5' targeting sequences in vivo (Xie et al. 2015), the tRNA-sgRNA fusion strategy effectively facilitates the expression of the sgRNAs fully matched to their targets, thus ensuring the activities of high-specificity Cas9. To more extensively evaluate the tRNA-sgRNA fusion strategy, we generated seven constructs harboring EC1fp:Cas9 and one of the seven types of double-sgRNA cassettes (Fig. 4). When we fused tRNA-Met instead of tRNA-Gly to the sgRNA targeting the TYR and $C P C$ genes, we obtained higher editing efficiencies (Fig. 4): $3.7 \%$ (pE-T\&C) versus $2.1 \%$ (pT\&CE), $4.2 \%$ (pE-T\&C2) versus $2.8 \%$ (pT\&C-E2), and $2.8 \%$ (pT\&C-E2) versus $2.3 \%$ (pT\&C-E2b). These results suggest that tRNA-Met is efficient and could be used together with tRNA-Gly for multiplex genome editing. The results also verified that the sgRNAs fully matched to targets conferred slightly higher editing efficiencies than the sgRNAs with the first base mismatched to the targets: $2.8 \%$ (pT\&C-E2) versus $2.1 \%$ (pT\&C-E) and $4.2 \%(\mathrm{pE}-\mathrm{T} \& \mathrm{C} 2)$ versus $3.7 \%$ (pE-T\&C).

Nevertheless, the above tRNA-sgRNA fusion strategies conferred significantly lower efficiencies than the general
$T R Y$ and $C P C$ genes of T1 plants are indicated. All the sgRNA target sequences in the five constructs were the same, and alignment of the target sequence with its target gene (TRY and $C P C)$ is indicated in Fig. 1 (sgR-T\&C)

strategy we previously reported $(>10 \%)$. We postulated that two tandem tRNA-sgRNA cassettes affected the expression of sgRNA. Therefore, we used two polymerase III promoters to drive the two tRNA-sgRNA cassettes, respectively. The results indicated that this strategy was more efficient than the former: $4.7 \%$ (pT\&C-E3a) versus $2.8 \%$ (pT\&C-E2). We then used the mutant sgRNA scaffold to replace the original one and obtained a significantly higher editing efficiency (13.4\%). To further provide evidence for the efficacy of the tRNA-sgRNA fusion strategies, we first generated two vectors, each harboring one tRNA-sgRNA targeting the ETC2 gene (Fig. S1). The results proved that upon fusion to tRNA, the mutant sgRNA scaffold conferred higher editing efficiency than the original $[9.2 \%(\mathrm{pEd})$ versus $5.2 \%(\mathrm{pEc})]$. The results also suggested that the off-target effects would be aggravated when the concentrations of sgRNA were significantly increased or when the mismatched base was changed to a matched one, regardless of whether the mismatch involved the first base (Fig. S1). Second, we generated four additional vectors, each harboring one of the two Cas9 cassettes and one of the three sgRNA cassettes targeting the BRII gene. The results similarly indicated that tRNA-sgR(m) conferred a significantly higher editing efficiency than tRNA-sgR(o), where $\operatorname{sgR}(\mathrm{m} / \mathrm{o})$ represents the sgRNA with the mutant or original scaffold, respectively, showing $12.4 \%(\mathrm{pBd})$ versus $1.3 \%(\mathrm{pBc})$ for the $\mathrm{T} 1$ mutant plants with observable phenotypes (Fig. S2).

In summary, our results suggest that depending on the target sites, the editing efficiencies of different tRNA-sgRNA fusion strategies could be largely described in decreasing order as follows: $\mathrm{tRNA}$ (Met) $-\mathrm{sgRm} \geq \mathrm{tRNA}$ (Gly) $-\operatorname{sgR}(m) \geq \operatorname{sgR}(m) \geq \operatorname{sgR}(0) \geq$ tRNA-sgR(o), where the $\operatorname{sgR}(\mathrm{m} / \mathrm{o})$ represents $\operatorname{sgRNAs}$ with a mutant or origina 1 scaffold, respectively. 

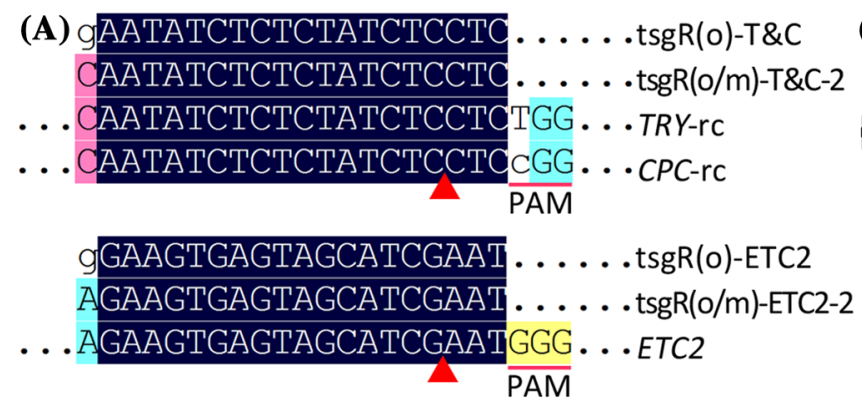

(C)

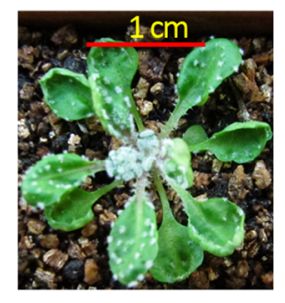

(D)

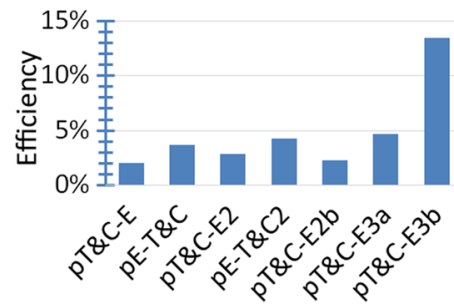

Fig. 4 Fusion of tRNA with mutant sgRNA scaffold conferred significantly higher efficiency than with the original sgRNA scaffold. a Alignment of the sgRNAs with their target genes. Only aligned regions of interest are displayed. -rc, reverse complement. b Two strategies were used to express two tRNA-sgRNA cassettes, one using a Pol3 promoter and another using two Pol3 promoters to drive two fused tRNA-sgRNA cassettes. Seven combinations of two tRNA genes and two sgRNA scaffolds were used in testing mutation effi-

\section{Validation of eight high-specificity SpCas9 variants}

High-specificity SpCas9 variants were developed based on two different strategies, namely, introducing mutations to weaken Cas9 binding to either the non-complementary or complementary DNA strand. These two strategies increase the stringency of guide RNA-DNA complementation for nuclease activation and therefore significantly improve editing specificity (Kleinstiver et al. 2016; Slaymaker et al.
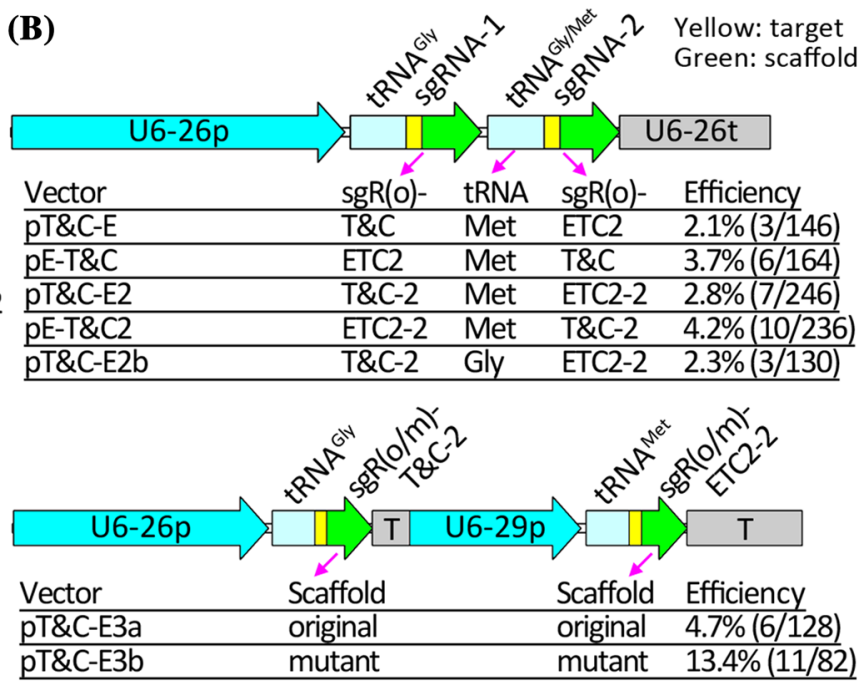

GTTIOAGAGCTATGCTGGAAACAGCATAGCAAGTTGAAATAM GTTTTAGAGCTA . . . . GAAA . . . . TAGCAAGTIAAAATAO

ciencies based on the two strategies. The alignment of the original (O) and mutant sgRNA (M) scaffolds are indicated and only regions with differences are displayed. Mutation efficiencies were calculated based on the ratio of the number of mutant plants with clustered leaf trichomes to the total number of $\mathrm{T} 1$ plants. $\mathbf{c}$ Representative phenotypes of mutants with clustered leaf trichomes. d Graphic comparison of mutation efficiencies induced by the seven constructs

2016). We generated eight SpCas9 variants of the maize codon-optimized Cas 9 using single or combinatory forms of the two strategies (Table 1). To evaluate the editing efficiencies of the eight Cas9 variants, we used the EC1.2-EC1.1 fusion promoter (EC1fp) to drive the Cas9 variants and the tRNA-sgR(m) strategy for targeting.

We first validated the editing efficiency of two reporter genes, $C P C$ and $T R Y$, which exhibit observable clustered leaf trichomes when simultaneously mutated. The results
Table 1 Mutant SpCas9 variants with enhanced specificity

\begin{tabular}{llllllllll}
\hline Cas9 variant & \multicolumn{9}{l}{ Mutated amino acids in the mutant SpCas9 variants } \\
\cline { 2 - 9 } & N497A & R661A & Q695A & K810A & K848A & Q926A & K1003A & R1060A \\
\hline eSpCas9(1.0) & $/$ & $/$ & $/$ & $\sqrt{ }$ & $/$ & $/$ & $\sqrt{ }$ & $\sqrt{ }$ \\
eSpCas9(1.1) & $/$ & $/$ & $/$ & $/$ & $\sqrt{ }$ & $/$ & $\sqrt{ }$ & $\sqrt{ }$ \\
eSpCas9(1.2) & $/$ & $/$ & $/$ & $\sqrt{ }$ & $\sqrt{ }$ & $/$ & $\sqrt{ }$ & $\sqrt{ }$ \\
SpCas9-HF1 & $\sqrt{ }$ & $\sqrt{ }$ & $\sqrt{ }$ & $/$ & $/$ & $\sqrt{ }$ & $/$ & $/$ \\
HF1-e(1.1) & $\sqrt{ }$ & $\sqrt{ }$ & $\sqrt{ }$ & $/$ & $\sqrt{ }$ & $\sqrt{ }$ & $\sqrt{ }$ & $\sqrt{ }$ \\
HF1a-e(1.1) & $\sqrt{ }$ & $\sqrt{ }$ & $\sqrt{ }$ & $/$ & $\sqrt{ }$ & $/$ & $\sqrt{ }$ & $\sqrt{ }$ \\
HF1b-e(1.2) & $/$ & $/$ & $/$ & $\sqrt{ }$ & $\sqrt{ }$ & $\sqrt{ }$ & $\sqrt{ }$ & $\sqrt{ }$ \\
HF1a-e(1.2) & $\sqrt{ }$ & $\sqrt{ }$ & $\sqrt{ }$ & $\sqrt{ }$ & $\sqrt{ }$ & $/$ & $\sqrt{ }$ & $\sqrt{ }$ \\
\hline
\end{tabular}

Numbers indicate the positions of amino acids at SpCas9, and letters before and after the numbers represent original and mutated amino acids, respectively. Slash or check marks indicate the absence or presence of a mutation, respectively. Five novel mutant $\operatorname{SpCas} 9$ variants, eSpCas9(1.2), HF1-e(1.1), HF1a-e(1.1), HF1b-e(1.2), and HF1a-e(1.2) were generated in this study 
indicated that only 1 of the 8 Cas 9 variants displayed mutations in the T1 plants, with an editing efficiency of $0.64 \%$ (1/156), significantly lower than that (17.3\%, 27/156) of the wild-type SpCas9 (Table S3). We then tested the editing efficiency of the T2 plants. The results demonstrated that the four SpCas9 variants, which were generated using combinations of mutations in two types of high-specificity SpCas9 variants, still conferred no observable mutations in the $\mathrm{T} 2$ plants (Table S3). The results also indicated that SpCas9-HF1 conferred significantly lower editing efficiency than eSpCas9 variants, of which $\mathrm{eSp}$ Cas9(1.1) exhibited the highest editing efficiency (Fig. 5). Interestingly, eSpCas9(1.2), the combinatory forms of
(A)

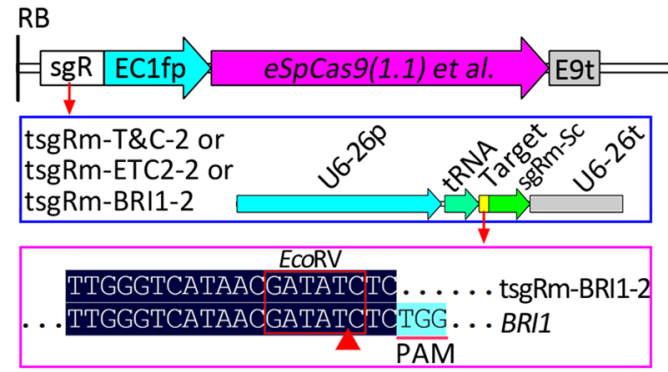

(C)

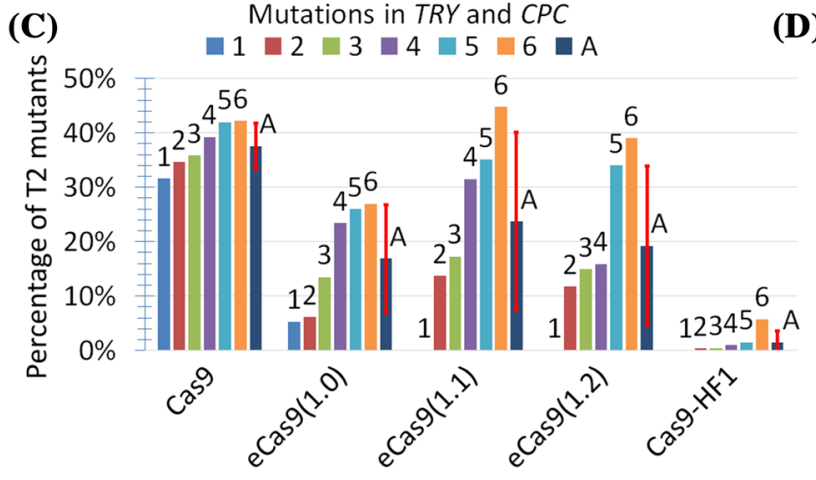

(E)

Mutations in BR/1

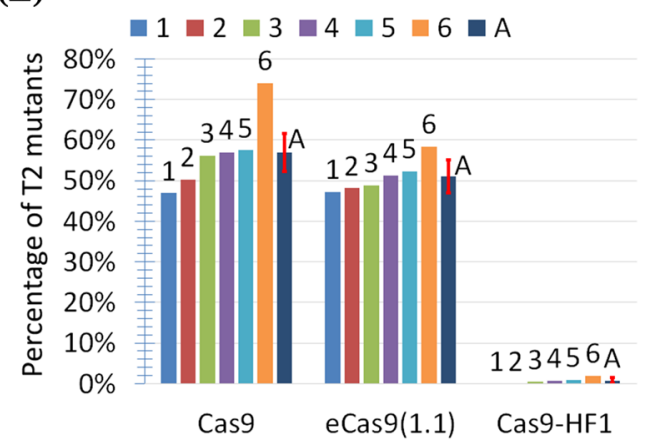

(D)
LB

(B)

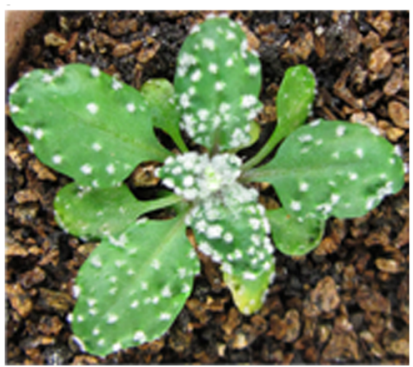

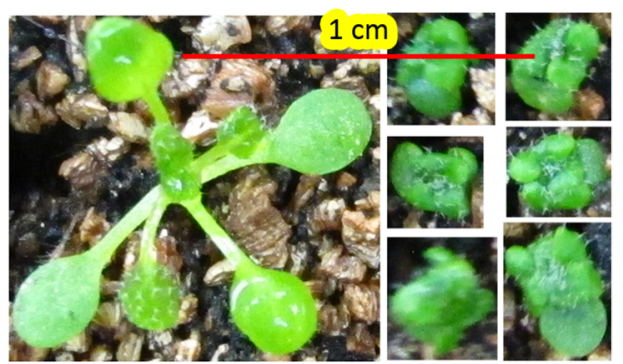

Mutations in ETC2

(F) $\square 1 \square 2 \square 3 \square 4 \square 5 \square 6 \square \mathrm{A}$

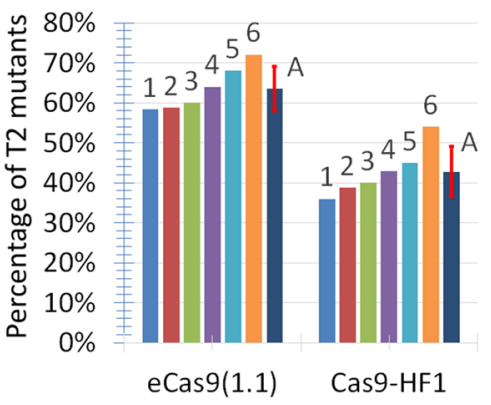

Fig. 5 Comparison of efficiencies of mutant $\operatorname{SpCas} 9$ variants with high specificity. a Structures of T-DNA and the sgRNA expression cassettes for testing the efficiencies of $\operatorname{Sp} \operatorname{Cas} 9$ variants. Alignment of one sgRNA with its target gene BRII is indicated, whereas the alignment of the other two sgRNAs with their target genes was described elsewhere. Only aligned regions of interest are displayed. b Phenotypes of a representative $\mathrm{T} 2$ mutant plant harboring tsgRm-T\&C-2 and $e \operatorname{SpCas} 9(1.1)$. c Efficiencies of mutations in the TRY and CPC genes of T2 plants, each harboring tsgRm-T\&C-2 and one of five SpCas 9 variants. Six T1 lines (\#1-6) with normal phenotypes were randomly selected for the analysis of mutations in the TRY and $C P C$ genes of the $\mathrm{T} 2$ population according to clustered leaf trichomes. A, average value of the six lines. d Phenotypes of representative bril mutants. One $\mathrm{T} 2$ plant with normal phenotypes and six segregated bril mutants are displayed. The T2 transgenic plants harboring $e S p$ $\operatorname{Cas} 9(1.1)$ were from the same pot and the same photo. e Efficiencies of mutations in BRII of the T2 plants harboring tsgR-BRI1-2 and one of three $\operatorname{SpCas} 9$ variants. Six T1 lines (\#1-6) with normal phenotypes were randomly selected for analysis of mutations in BRI1 of their T2 populations according to the dwarf phenotypes. A, average value of the six lines. $\mathbf{f}$ Efficiencies of mutations in ETC2 of the T2 plants, each harboring tsgR-ETC2-2 and one of the two mutant $S p C a s 9$ variants. Six T1 lines (\#1-6) without mutations were randomly selected for analysis of mutations in ETC2 gene of their T2 population by direct sequencing of PCR products. $A$ average value of the six lines 
eSpCas9(1.0)/(1.1), conferred high editing efficiency that was comparable to that in eSpCas9(1.0) (Fig. 5).

To further compare the editing efficiencies of eSpCas9(1.1) and SpCas9-HF1, we tested two additional target genes, namely, BRI1 and ETC2 (Fig. 5). No mutations in $B R I 1$ of T1 plants harboring eSpCas9(1.1) or SpCas9-HF1 were observed (Table S4). We identified eSpCas9(1.1)induced mutations in the ETC2 gene of the T1 plants, with an efficiency of $2.4 \%$ (1/42), significantly lower than that $(9.2 \%, 9 / 98)$ of the wild-type SpCas9 (Table S5). For T2 plants, eSpCas9(1.1) conferred significantly higher editing efficiency of mutations in BRII or ETC2 than SpCas9-HF1. Finally, no off-target mutations induced by eSpCas9(1.1) were detected, indicating that eSpCas9(1.1) was indeed highly specific (Table S6).

Overall, our results indicated that depending on the target sites, the editing efficiencies in the $\mathrm{T} 1$ plants of the eight high-specificity SpCas 9 could be largely described in decreasing order as follows: SpCas9 > > eSpCas9(1.1) > > the others, whereas those in the $\mathrm{T} 2$ plants could be largely described in decreasing order as follows: $\mathrm{SpCas} 9 \geq \mathrm{eSp}-$ Cas9(1.1) > eSpCas9(1.0) $\geq \operatorname{eSpCas9(1.2)~>>~SpCas9-~}$ HF1 $>>$ the others. These results suggest that high-specificity SpCas9 variants, particularly SpCas9-HF1, require significantly higher expression levels for their on-target editing efficiency. These results also suggest that the highspecificity SpCas9 variants driven by constitutive and strong promoters, in combination with tRNA-sgRNA(m) fusion strategy, enable highly efficient genome editing in crops. Editing efficiency could be further increased when the high specificity CRISPR/Cas9 systems are combined with geminivirus-based replicon systems (Cermak et al. 2017).

\section{High-frequency T-DNA insertions into cleavage sites of CRISPR/Cas9 targets}

Because we were unable to obtain the $C P C$ fragment of line \#4 by PCR amplification, we then investigated the possibility of T-DNA insertions into the cleavage sites (Fig. 6). As expected, the cleavage sites of the two $C P C$ alleles were inserted by two T-DNAs, respectively (Fig. 3). The juncture sequences between the upstream $C P C$ of the cleavage site and the left border of the T-DNA were identical for the two alleles, whereas those between the downstream $C P C$ of the cleavage site and the right border of T-DNA differed between the two alleles (Fig. 6).

We also investigated $30 \mathrm{~T} 1$ etc 2 mutant lines generated with the three different constructs that targeted the ETC2 gene for the possibility of T-DNA insertions into the cleavage site. The results indicated that depending on
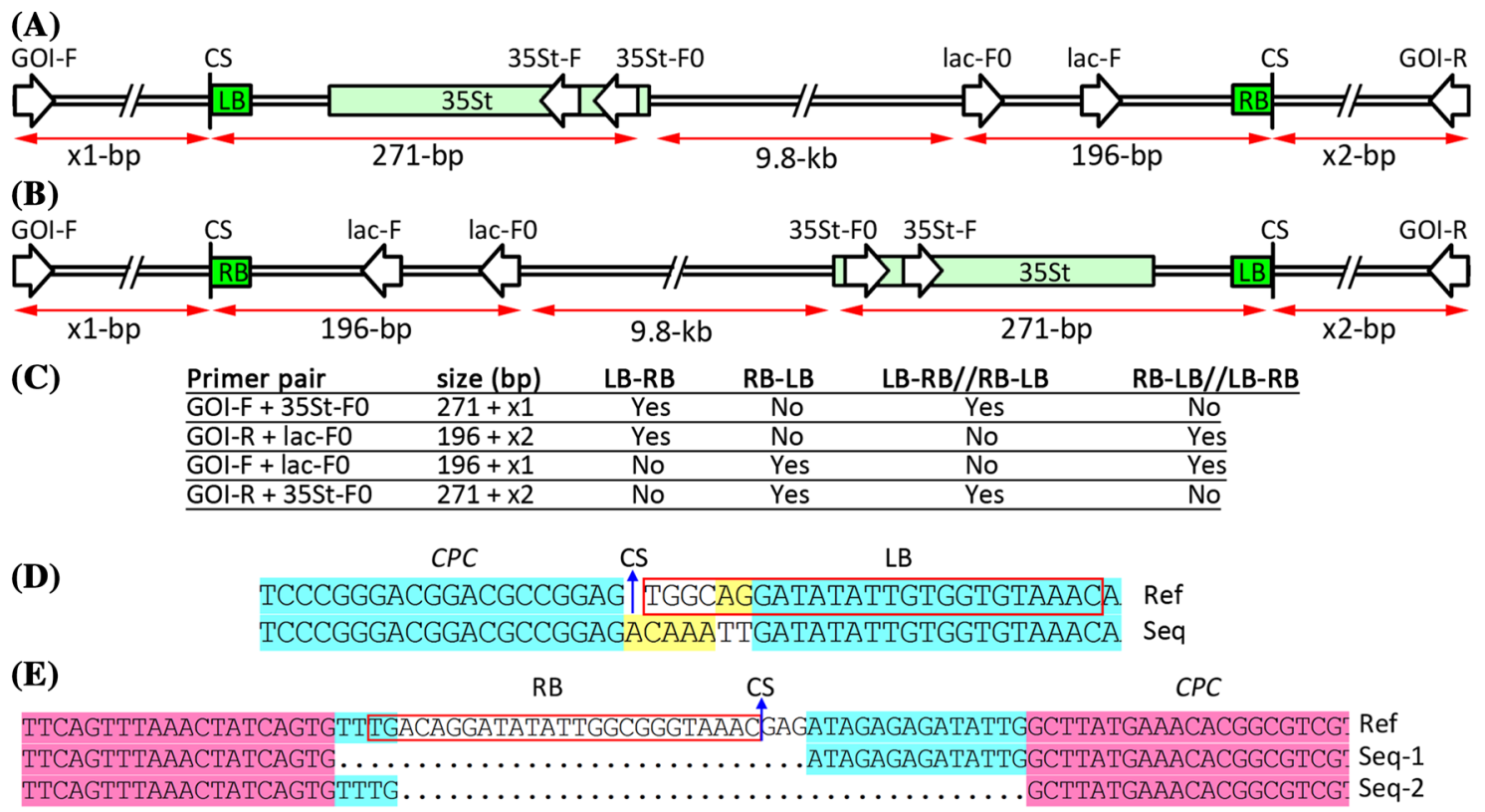

Fig. 6 Failure in PCR amplification can be attributed to CRISPR/ Cas9-mediated T-DNA insertions into the cleavage sites. a-c Schematic diagram for the identification of T-DNA insertions into a cleavage site of the CRISPR/Cas9 system. The possibly forward or reverse T-DNA insertions with one, two, or multiple copies of T-DNA, and the sizes of the PCR fragments with the indicated primer pairs are summarized. GOI, gene of interest. CS, cleavage site. -F/-R, forward/ reverse primer. LB/RB, left/right border of T-DNA. 35St, CaMV 35S terminator. d, e Juncture sequences between the T-DNA and $C P C$ sequences before (d) or behind (e) the cleavage site of CRISPR/Cas9 in \#4 T1 lines. Ref reference sequence of the junctures, Seq actual sequence from sequencing. Dots represent deletions. Note: Two types of juncture sequences between the RB and the $C P C$ gene indicate that two copies of T-DNA were inserted into the two alleles, which accounted for the failure in PCR 
Cas9 promoters, the frequencies of targeted T-DNA insertions were $6.7 \%(1 / 15), 83.3 \%(5 / 6)$, and $77.8 \%(7 / 9)$ for the three constructs, respectively (Fig. S3). In addition, we encountered the same problem for the analysis of mutations in the $H A B 1$ gene of line \#4 T1 and T2 plants in our previous study (Zhang et al. 2017b). This failure in PCR amplifications may also be due to T-DNA insertions. As expected, we obtained juncture sequences between the downstream $H A B 1$ gene of the cleavage site and the T-DNA, and therefore confirmed the T-DNA insertions (Fig. S4). We then analyzed the T-DNA insertions in the other 16 lines, revealing that T-DNA was inserted into the $H A B 1$ gene of the two additional lines, \#1 and \#14 (Fig. S4).

$\mathrm{SaCas} 9$ requires a longer PAM than $\mathrm{SpCas} 9$ for target recognition, and therefore may be utilized in reducing off-target mutations. To validate our maize codon-optimized SaCas9, we generated a binary vector harboring EC1f:SaCas9 and U6:sgRNA. We tested a previously reported $A D H 1$ target (Steinert et al. 2015) and found that 15\% (15/100) of the T1 plants harbored homozygous or biallelic mutations in the $A D H 1$ gene, indicating that the egg cell-specific promotercontrolled SaCas9 system had a similar efficiency to that of SpCas9 (Fig. S5). Analysis of T-DNA insertions also indicated that $47 \%(7 / 15)$ of the $\mathrm{T} 1$ mutant lines harbored T-DNA insertions at the SaCas9 cleavage site of the $A D H 1$ gene (Fig. S5).

\section{Discussion}

CRISPR/Cas9 specificity is affected by various factors, including features of off-target sites and effective concentrations of the Cas9/sgRNA complexes. Determination of CRISPR/Cas9 specificity also depends on assay methods, either biased or unbiased, and in silico off-target prediction algorithms. This complexity is regarded as a confounding factor for off-target mutation assays and the use of different standards for measuring and reporting off-target activity affects the preciseness of conclusions (Tsai and Joung 2016; Tycko et al. 2016; Wu et al. 2014).

CRISPR/Cas9 is known to be significantly more specific in plant cells than in human cells (Feng et al. 2014; Peterson et al. 2016; Wolt et al. 2016; Zhang et al. 2014). Two reports based on whole genome sequencing (WGS) or deep sequencing provide evidence that the editing efficiency of the CRISPR/Cas9 system is highly specific in Arabidopsis (Feng et al. 2014; Peterson et al. 2016). In one of these reports, deep sequencing of a total of 178 off-target sites demonstrated that the high specificity of CRISPR/Cas9 in Arabidopsis and the low expression levels of the Cas9 driven by the UBQ10 promoter were hypothesized to be the reason for the undetectable off-target events (Peterson et al. 2016). Actually, competitive binding of 14 sgRNA variants to Cas 9 definitely led to significantly lower effective concentrations of each Cas9/sgRNA complex variant. Because out of the 14 sgRNAs, two (CLE18_2 and GLV8_1) did not show any evidence of on-target editing, the 26 off-target sites from these two sgRNAs could be excluded from the list of offtarget sites. Thus, out of the rest of the 152 off-target sites from the 12 sgRNAs, 83\% (126/152) and 17\% (26/152) harbored 4 and 3 mismatches, respectively, indicating that the $12 \mathrm{sgRNAs}$ were predicted to be specific by in silico analysis (Peterson et al. 2016). Therefore, lower effective concentrations of each Cas9/sgRNA complex variant and relatively specific sgRNAs might be the reasons for the reported high specificity of CRISPR/Cas9 in Arabidopsis.

In the other report, in-depth WGS of two T1 plants (\#T146 and \#T1-55) and one T2 plant (\#T2-46) harboring GAIsgRNA1 showed no indication of any off-target events in the potential off-target sites harboring 1-4 mismatches (Feng et al. 2014). However, although WGS is ideal for off-target mutation assays of individual plants, due to its high cost, it is not practical to systematically assess a large number of plants and sgRNA variants to determine Cas 9 specificity (Wu et al. 2014). Thus, most low-frequency off-target events would go unaccounted (Wu et al. 2014). Although approximately $60 \mathrm{~T} 1$ plants harboring GAI-sgRNA1 were examined for off-target mutations by PCR followed by sequencing, a large number of T2 plants were not examined. Because only one sgRNA and a limited number of plants from limited generations were investigated for off-target mutations, the data cannot rule out the possibility of low-frequency off-target events induced by the same sgRNA and high-frequency offtarget events induced by other sgRNA variants (Feng et al. 2014).

Based on the above analysis of the results in the two reports, it is not abnormal that we observed high-frequency off-target mutations in Arabidopsis in our study (Figs. 1, 2), although the sgRNA was predicted to have a high specificity score (Haeussler et al. 2016, Hsu et al. 2013). Actually, the observations in this study were also inconsistent with those in our previous reports, wherein we had suggested that the same sgRNA was specific (Wang et al. 2015; Xing et al. 2014). The main reason for this inconsistency in results is that our previous conclusions were based on only one or two T1 plants, and that different promoters were used to drive Cas9. Consistent with this notion, in our previous report on sgRNA targeting the $A B I 1$ gene, the investigation of eight T1 abil mutants and two T2 populations for offtarget mutations generated similar results to that observed in the present study (Zhang et al. 2017b). Out of the $8 \mathrm{~T} 1$ abil mutants, 1 (1/8 or $13 \%) \mathrm{T} 1$ plant harbored heterozygous or chimeric off-target mutations in AT5G02760 with 2 mismatches, and all the 8 lines harbored no off-target mutations in $A B I 2$ with only 1 mismatch and the other 2 genes with 3 mismatches. Analysis of 52 T2 plants from the T1 line 
harboring off-target mutations in AT5G02760 showed that $85 \%$ of the T2 (44/52) plants harbored off-target mutations in $A T 5 G 02760$, and 17\% (9/52) harbored off-target mutations in $A B I 2$ and both genes. Analysis of $41 \mathrm{~T} 2$ plants from the other $\mathrm{T} 1$ line harboring no off-target mutations indicated that $10 \%$ of the T2 (4/41) plants harbored off-target mutations in AT5G02760, 4.9\% (2/41) harbored off-target mutations in $A B I 2$, and $2.4 \%$ (1/41) harbored off-target mutations in both genes (Zhang et al. 2017b). Consistent with the results in the present study (Fig. 2), these findings suggest that offtarget effects are aggravated in the next generation. It will be interesting to use our egg cell-specific promoter-controlled (EPC) CRISPR/Cas9 system to determine the specificity of GAI-sgRNA1 since its specificity score was 64 relative to the range of $0-100$, much lower than that (94) of the sgRETC2 (Haeussler et al. 2016, Hsu et al. 2013).

The high-frequency off-target mutations in the $C P C$ gene could be attributed to some sequence features such as position, distribution, and identity of mismatches (Tsai and Joung 2016; Wu et al. 2014). Although the off-target site in the $C P C$ gene has three mismatches with the sgRNA-ETC2, the first mismatch located at the first base distal to PAM is usually tolerated by the CRISPR/Cas9 system (Fig. 1). The second mismatch is also far away from the PAM and/or the two mismatches are situated far from each other, which may also account for the high-frequency off-target mutations in the $C P C$ gene rather than the TRY gene or AT5G50230 (Fig. 1). The PAM-proximal 11-nt were defined as the seed region for Cas 9 cutting activity and mismatches in the region are less tolerated (Wu et al. 2014). In some other assays, the seed region was narrowed down to PAM-proximal 5-nt (Wu et al. 2014). Different concentrations of the CRISPR/ Cas9 complex and duration of Cas9 binding and cleavage may be responsible for the observed variations in the length of the seed detected by different assays. Therefore, it is not strange for the high-frequency off-target mutations in the $C P C$ off-target site harboring a mismatch in the 8th nt in the PAM-proximal position (Fig. 1). Similar to this study, our previous investigation involving sgRNA-ABI1 indicated that the off-target sites also harbor a mismatch in the 9th nt in the PAM-proximal position (Zhang et al. 2017b). The frequency of off-target events was also affected by mismatch identity and could be largely indicated as: $\mathrm{rN}: \mathrm{dT} \geq \mathrm{rU}: \mathrm{dG}>>\mathrm{rC}: \mathrm{dC}>>\mathrm{rA} / \mathrm{rG}: \mathrm{dA} / \mathrm{dG}$ (Doench et al. 2016; Tsai and Joung 2016; Tycko et al. 2016). The 8th and 9th mismatches mentioned earlier were all of the $\mathrm{rC}: \mathrm{dT}$ mismatch, suggesting that this mismatch identity was frequently tolerated and contributed to the off-target effects in plants. The observation that the off-target site harboring the two mismatches to the sgRNA targeting $A B I 1$ displayed higher frequency off-target effects than that harboring one mismatch (Zhang et al. 2017b) indicates that more factors should be considered in the development of more precise off-target prediction algorithms that are based on large training data sets from high-throughput experiments.

Because our EPC system is a relatively short-time expression system (Wang et al. 2015), it seemed that it should have a significantly lower off-target frequency than systems driven by constitutive promoters, including $2 \times 35 \mathrm{~S}$, UBQ1, UBQ10, and PcUbi4-2 (Fauser et al. 2014; Feng et al. 2014; Peterson et al. 2016). On the contrary to this supposition, we observed high-frequency off-target mutations in the $C P C$ gene, which suggests that in the EPC system, a high dosage of the CRISRP/Cas9 complex in egg cells and one cell-stage embryos compensated the short duration for off-target mutations. Consistent with this notion, comparison of the results obtained with tRNA-sgRNA(m) and tRNA-sgRNA(o) suggested that the increased dosage of the complex significantly enhanced the frequency of off-target mutations: 66.7 versus $20.0 \%$ (Fig. S1).

It is comprehensible that tRNA-sgRNA(m) generally has a significantly higher editing efficiency than tRNAsgRNA(o) (Xie et al. 2015) and sgRNA(m) (Dang et al. 2015). However, the present study showed that tRNA$\operatorname{sgRNA}(0)$ had a markedly lower editing efficiency than $\operatorname{sgRNA}(0)$, quite contrary to our anticipation (Figs. 3, 4, S1 and S2). This finding may be attributed to three aspects. First, the EPC system might be much more sensitive to fluctuations in effective concentrations or activities of the CRISPR/Cas 9 complex than systems driven by constitutive promoters. Consistent with this notion, different terminators (Wang et al. 2015) and even the mCherry cassette behind the terminator (Fig. 3) significantly affected the editing efficiency of the EPC system. In addition, high-specificity SpCas9 variants induced lower efficiency mutations in the T1 plants than their wild-type counterpart, indicating that the EPC system was more sensitive to the fluctuation in the activities of the CRISPR/Cas9 variants. For constitutively expressed CRISPR/Cas9 systems, low levels of sgRNA, if existing, from tRNA-sgRNA(o) could be compensated by the extended duration of expression or activity of the complex, thus leading to an overall high editing efficiency (Xie et al. 2015). Second, the U6 promoters we used might be more sensitive to $4 \times \mathrm{T}$, a potential terminator of Pol-III promoters in the original sgRNA scaffold than the OsU3 promoter. Third, the tRNA secondary structure (the cloverleaf structure) formed after transcription might enhance termination at the $4 \times \mathrm{T}$ sites. Whatever the reason, the present study observed that the tRNA-sgRNA(m) was the optimal form that facilitated the successful application of not only the mCherry cassette in counter-selection of Cas9-free plants (Gao et al. 2016), but also high-specificity Cas 9 variants (Chen et al. 2017; Kleinstiver et al. 2016; Slaymaker et al. 2016) in avoiding the occurrence of off-target effects.

Our results also suggest that high-specificity SpCas9 mutant variants require much higher concentrations to 
maintain high editing efficiency than the wild-type counterpart (Fig. 5), although it remains to be determined whether the mutant sgRNA scaffold affected their editing efficiencies. Driven by constitutive and strong promoters in combination with the tRNA-sgRNA(m) fusion strategy, these SpCas9 variants can be used for high-specificity and high-efficiency genome editing in crops. Particularly in geminivirus-mediated CRISPR/Cas9 systems, the editing efficiency of highspecificity SpCas9 will be greatly strengthened since DNA replicons harboring Cas9 and sgRNA cassettes transiently amplify hundreds of copies in a plant cell, thus leading to very high concentrations of the CRISPR/Cas9 complex in a cell (Cermak et al. 2017). Hence, together with the rapid evolution of integrated applications of geminivirus-based replicon systems and CRISPR/Cas9 systems, high-specificity SpCas9 will be particularly useful in avoiding off-target mutations caused by high-dosage CRISPR/Cas 9 complexes in a cell (Gil-Humanes et al. 2017; Wang et al. 2017).

One of our unexpected but interesting findings in this report was the high-frequency T-DNA insertions into cleavage sites (Figs. 6, S3-S5). This finding could facilitate the experimental design for targeted integration of transgenes (Li et al. 2016; Salomon and Puchta 1998; Tzfira et al. 2003) and may trigger additional concerns for mutation analysis. First, when encountering a failure in PCR amplification of a target region, T-DNA insertions should be considered as a possibility. Second, in previous reports, the mutation types that were identified as insertions of unknown large fragments might be re-considered as T-DNA insertion events. Third, for T1 Arabidopsis mutants generated using the EPC system or T0 mutants generated from embryogenic callus, ratios of homozygous or biallelic mutants are subject to overestimation and underestimation, respectively. The targeted integrations of two copies of T-DNA into the two alleles of the $C P C$ gene in this report or the $H A B l$ gene in a previous study might also represent novel knowledge of Agrobacterium-mediated T-DNA insertions into plant genome. First, CRISPR/Cas9-mediated DNA cleavage could be completed before T-DNAs are integrated into the genome of target cells. Second, for Arabidopsis floral dip transformation (Desfeux et al. 2000), random integration of T-DNA usually occurs before fertilization, whereas CRISPR/Cas9-mediated targeted integration of T-DNAs occurs after fertilization.

Since the sgR-ETC2 induced off-target mutations in $C P C$ with high frequency, in $T R Y$ with medium frequency, and in AT5G50230 with low frequency under detectable level, our results suggest that carefully selected target sites largely guarantee high specificity. This observation was supported by our previous report, wherein we detected sgR-ABI1induced off-target mutations in ABI2 and AT5G02760, but did not detect off-target mutations in $A T 2 G 25070$ and $A T 3 G 17090$. The intended on-target sites should have no potential off-target sites that harbor less than three mismatches and easily tolerated mismatch features. In addition, awareness is advised when targeting multiple highly homologous genes because off-target sites are possibly adjacent to on-target sites, which may lead to enhanced off-target effects, similar to the case reported in this study. Overall, we recommend using combinatory forms of the following six strategies to avoid off-target effects in plants (Tycko et al. 2016). First, high-specificity targets should be carefully selected using in silico predictions (Haeussler et al. 2016). Second, Cas9-free mutants should be isolated as far as possible (Gao et al. 2016; Lu et al. 2017). Third, when necessary, high-specificity SpCas9 variants in combination with the tRNA-sgRNA(m) fusion method can be used (Chen et al. 2017; Kleinstiver et al. 2016; Kulcsar et al. 2017; Slaymaker et al. 2016; Xie et al. 2015; Zhang et al. 2017a). Fourth, when necessary, SaCas9 or other orthologs with higher specificity can be used (Steinert et al. 2015). Fifth, when necessary, paired nickases can be used (Fauser et al. 2014). Last, when necessary, DNA-free methods can be employed (Liang et al. 2017; Svitashev et al. 2016; Woo et al. 2015).

Collectively, this is the first report on the observations of high-frequency off-target mutagenesis induced by CRISPR/ Cas9 in plants; our results suggest that in plants harboring CRISPR/Cas9 components, continuous attention should be paid to off-target effects induced by CRISPR/Cas9 in current and subsequent generations, and that the tools optimized in this report will be useful to improve genome editing efficiency and specificity in plants and other organisms.

\section{Methods}

\section{Vector construction}

All primers used in this study are listed in Table S7. The cloning CRISPR/Cas9 binary vectors, the PCR template vectors, the final CRISPR/Cas9 binary vectors each harboring one sgRNA cassette, and the final CRISPR/Cas9 binary vectors each harboring two sgRNA cassettes are listed in Tables S8-11. Detailed descriptions of the vector construction and annotated sequences of the sgRNA cassettes for cloning are provided in Methods S1 and Appendix S1-S5, respectively.

\section{Generation of transgenic Arabidopsis plants and analysis of mutations and T-DNA insertions}

We transformed the 26 and 7 final CRISPR/Cas 9 binary vectors, harboring one and two sgRNA cassettes, respectively, into Agrobacterium strain GV3101. We generated transgenic plants by transformation of Arabidopsis Col-0 wild-type plants via the floral dip method. We screened the seeds collected from the transformed plants on MS plates 
supplemented with $25 \mathrm{mg} / \mathrm{L}$ hygromycin and transplanted the resistant seedlings ( $\mathrm{T} 1)$ to soil.

To analyze mutations, we extracted genomic DNA from $\mathrm{T} 1$ or $\mathrm{T} 2$ transgenic plants grown in soil. We amplified fragments surrounding on-target and adjacent off-target sites by PCR using gene-specific primers (Table S7). We submitted purified PCR products for direct sequencing with the corresponding primers. We cloned poorly sequenced PCR products and submitted individual positive clones for sequencing using the T7 and SP6 primers.

To analyze T-DNA insertions into the cleavage sites, we used the T-DNA LB/RB-specific primer 35St-F0/lac-F0 and the gene-specific primers (Table S7) for PCR amplifications of the juncture sequences between the T-DNA and target genes. We submitted the purified PCR products for direct sequencing with primers $35 \mathrm{St}-\mathrm{F} / \mathrm{lac}-\mathrm{F}$ (Table S7). We cloned poorly sequenced PCR products and submitted individual positive clones for sequencing using the $\mathrm{T} 7$ primer.

Acknowledgements This work was supported by Grants from the National Crop Breeding Fund (2016YFD0101804), the National Natural Science Foundation of China (31670371), the National Transgenic Research Project (2016ZX08009002), the Chinese Universities Scientific Fund (2017TC007), and Collaborative Innovation Center of Crop Stress Biology, Henan Province.

Author Contributions QJC planned and designed the research. QZ, HLX, ZPW, HYZ and FY conducted experiments and analyzed the data. QJC and XCW wrote the paper.

\section{Compliance with ethical standards}

Conflict of interest The authors declare no conflict of interest.

Open Access This article is distributed under the terms of the Creative Commons Attribution 4.0 International License (http://creativeco mmons.org/licenses/by/4.0/), which permits unrestricted use, distribution, and reproduction in any medium, provided you give appropriate credit to the original author(s) and the source, provide a link to the Creative Commons license, and indicate if changes were made.

\section{References}

Carroll D (2013) Staying on target with CRISPR-Cas. Nat Biotechnol 31:807-809

Carroll D, Charo RA (2015) The societal opportunities and challenges of genome editing. Genome Biol 16:242

Cermak T, Curtin SJ, Gil-Humanes J, Cegan R, Kono TJY, Konecna E, Belanto JJ, Starker CG, Mathre JW, Greenstein RL, Voytas DF (2017) A Multipurpose toolkit to enable advanced genome engineering in plants. Plant Cell 29:1196-1217

Chen JS, Dagdas YS, Kleinstiver BP, Welch MM, Sousa AA, Harrington LB, Sternberg SH, Joung JK, Yildiz A, Doudna JA (2017) Enhanced proofreading governs CRISPR-Cas9 targeting accuracy. Nature 550:407-410
Cong L, Ran FA, Cox D, Lin S, Barretto R, Habib N, Hsu PD, Wu X, Jiang W, Marraffini LA, Zhang F (2013) Multiplex genome engineering using CRISPR/Cas systems. Science 339:819-823

Dai X, Zhang Y, Zhang D, Chen J, Gao X, Estelle M, Zhao Y (2015) Embryonic lethality of Arabidopsis abp1-1 is caused by deletion of the adjacent BSM gene. Nat Plants 1:15183

Dang Y, Jia G, Choi J, Ma H, Anaya E, Ye C, Shankar P, Wu H (2015) Optimizing sgRNA structure to improve CRISPR-Cas9 knockout efficiency. Genome Biol 16:280

Desfeux C, Clough SJ, Bent AF (2000) Female reproductive tissues are the primary target of Agrobacterium-mediated transformation by the Arabidopsis floral-dip method. Plant Physiol 123:895-904

Doench JG, Fusi N, Sullender M, Hegde M, Vaimberg EW, Donovan KF, Smith I, Tothova Z, Wilen C, Orchard R, Virgin HW, Listgarten J, Root DE (2016) Optimized sgRNA design to maximize activity and minimize off-target effects of CRISPR-Cas9. Nat Biotechnol 34:184-191

Fauser F, Schiml S, Puchta H (2014) Both CRISPR/Cas-based nucleases and nickases can be used efficiently for genome engineering in Arabidopsis thaliana. Plant J 79:348-359

Feng Z, Mao Y, Xu N, Zhang B, Wei P, Yang DL, Wang Z, Zhang Z, Zheng R, Yang L, Zeng L, Liu X, Zhu JK (2014) Multigeneration analysis reveals the inheritance, specificity, and patterns of CRISPR/Cas-induced gene modifications in Arabidopsis. Proc Natl Acad Sci USA 111:4632-4637

Fu Y, Foden JA, Khayter C, Maeder ML, Reyon D, Joung JK, Sander JD (2013) High-frequency off-target mutagenesis induced by CRISPR-Cas nucleases in human cells. Nat Biotechnol 31:822-826

Gao Y, Zhang Y, Zhang D, Dai X, Estelle M, Zhao Y (2015) Auxin binding protein 1 (ABP1) is not required for either auxin signaling or Arabidopsis development. Proc Natl Acad Sci USA 112:2275-2280

Gao X, Chen J, Dai X, Zhang D, Zhao Y (2016) An effective strategy for reliably isolating heritable and Cas9-free Arabidopsis mutants generated by CRISPR/Cas9-mediated genome editing. Plant Physiol 171:1794-1800

Gil-Humanes J, Wang Y, Liang Z, Shan Q, Ozuna CV, Sanchez-Leon S, Baltes NJ, Starker C, Barro F, Gao C, Voytas DF (2017) Highefficiency gene targeting in hexaploid wheat using DNA replicons and CRISPR/Cas9. Plant J 89:1251-1262

Haeussler M, Schonig K, Eckert H, Eschstruth A, Mianne J, Renaud JB, Schneider-Maunoury S, Shkumatava A, Teboul L, Kent J, Joly JS, Concordet JP (2016) Evaluation of off-target and on-target scoring algorithms and integration into the guide RNA selection tool CRISPOR. Genome Biol 17:148

Hsu PD, Scott DA, Weinstein JA, Ran FA, Konermann S, Agarwala V, Li Y, Fine EJ, Wu X, Shalem O, Cradick TJ, Marraffini LA, Bao G, Zhang F (2013) DNA targeting specificity of RNA-guided Cas9 nucleases. Nat Biotechnol 31:827-832

Jinek M, Chylinski K, Fonfara I, Hauer M, Doudna JA, Charpentier E (2012) A programmable dual-RNA-guided DNA endonuclease in adaptive bacterial immunity. Science 337:816-821

Kleinstiver BP, Pattanayak V, Prew MS, Tsai SQ, Nguyen NT, Zheng Z, Joung JK (2016) High-fidelity CRISPR-Cas9 nucleases with no detectable genome-wide off-target effects. Nature 529:490-495

Kulcsar PI, Talas A, Huszar K, Ligeti Z, Toth E, Weinhardt N, Fodor E, Welker E (2017) Crossing enhanced and high fidelity SpCas9 nucleases to optimize specificity and cleavage. Genome Biol $18: 190$

Li J, Meng X, Zong Y, Chen K, Zhang H, Liu J, Li J, Gao C (2016) Gene replacements and insertions in rice by intron targeting using CRISPR-Cas9. Nat Plants 2:16139

Liang Z, Chen K, Li T, Zhang Y, Wang Y, Zhao Q, Liu J, Zhang H, Liu C, Ran Y, Gao C (2017) Efficient DNA-free genome editing 
of bread wheat using CRISPR/Cas9 ribonucleoprotein complexes. Nat Commun 8:14261

Lu HP, Liu SM, Xu SL, Chen WY, Zhou X, Tan YY, Huang JZ, Shu QY (2017) CRISPR-S: an active interference element for a rapid and inexpensive selection of genome-edited, transgene-free rice plants. Plant Biotechnol J 15:1371-1373

Mali P, Yang L, Esvelt KM, Aach J, Guell M, DiCarlo JE, Norville JE, Church GM (2013) RNA-guided human genome engineering via Cas9. Science 339:823-826

O'Geen H, Yu AS, Segal DJ (2015) How specific is CRISPR/Cas9 really? Curr Opin Chem Biol 29:72-78

Peterson BA, Haak DC, Nishimura MT, Teixeira PJ, James SR, Dangl JL, Nimchuk ZL (2016) Genome-wide assessment of efficiency and specificity in CRISPR/Cas9 mediated multiple site targeting in Arabidopsis. PLoS ONE 11:e0162169

Salomon S, Puchta H (1998) Capture of genomic and T-DNA sequences during double-strand break repair in somatic plant cells. EMBO J 17:6086-6095

Slaymaker IM, Gao L, Zetsche B, Scott DA, Yan WX, Zhang F (2016) Rationally engineered Cas9 nucleases with improved specificity. Science 351:84-88

Steinert J, Schiml S, Fauser F, Puchta H (2015) Highly efficient heritable plant genome engineering using Cas9 orthologues from Streptococcus thermophilus and Staphylococcus aureus. Plant J 84:1295-1305

Svitashev S, Schwartz C, Lenderts B, Young JK, Mark Cigan A (2016) Genome editing in maize directed by CRISPR-Cas9 ribonucleoprotein complexes. Nat Commun 7:13274

Tsai SQ, Joung JK (2016) Defining and improving the genomewide specificities of CRISPR-Cas9 nucleases. Nat Rev Genet 17:300-312

Tycko J, Myer VE, Hsu PD (2016) Methods for optimizing CRISPRCas9 genome editing specificity. Mol Cell 63:355-370

Tzfira T, Frankman LR, Vaidya M, Citovsky V (2003) Site-specific integration of Agrobacterium tumefaciens T-DNA via doublestranded intermediates. Plant Physiol 133:1011-1023
Wang ZP, Xing HL, Dong L, Zhang HY, Han CY, Wang XC, Chen QJ (2015) Egg cell-specific promoter-controlled CRISPR/Cas9 efficiently generates homozygous mutants for multiple target genes in Arabidopsis in a single generation. Genome Biol 16:144

Wang M, Lu Y, Botella JR, Mao Y, Hua K, Zhu JK (2017) Gene targeting by homology-directed repair in rice using a geminivirus-based CRISPR/Cas9 system. Mol Plant 10:1007-1010

Wolt JD, Wang K, Sashital D, Lawrence-Dill CJ (2016) Achieving plant CRISPR targeting that limits off-target effects. Plant Genome. https://doi.org/10.3835/plantgenome2016.05.0047

Woo JW, Kim J, Kwon SI, Corvalan C, Cho SW, Kim H, Kim SG, Kim ST, Choe S, Kim JS (2015) DNA-free genome editing in plants with preassembled CRISPR-Cas9 ribonucleoproteins. Nat Biotechnol 33:1162-1164

Wu X, Kriz AJ, Sharp PA (2014) Target specificity of the CRISPRCas9 system. Quant Biol 2:59-70

Xie K, Minkenberg B, Yang Y (2015) Boosting CRISPR/Cas9 multiplex editing capability with the endogenous tRNA-processing system. Proc Natl Acad Sci USA 112:3570-3575

Xing HL, Dong L, Wang ZP, Zhang HY, Han CY, Liu B, Wang XC, Chen QJ (2014) A CRISPR/Cas9 toolkit for multiplex genome editing in plants. BMC Plant Biol 14:327

Yin K, Gao C, Qiu JL (2017) Progress and prospects in plant genome editing. Nat Plants 3:17107

Zhang H, Zhang J, Wei P, Zhang B, Gou F, Feng Z, Mao Y, Yang L, Zhang H, Xu N, Zhu JK (2014) The CRISPR/Cas9 system produces specific and homozygous targeted gene editing in rice in one generation. Plant Biotechnol J 12:797-807

Zhang D, Zhang H, Li T, Chen K, Qiu JL, Gao C (2017a) Perfectly matched 20-nucleotide guide RNA sequences enable robust genome editing using high-fidelity SpCas9 nucleases. Genome Biol 18:191

Zhang HY, Wang XH, Dong L, Wang ZP, Liu B, Lv J, Xing HL, Han CY, Wang XC, Chen QJ (2017b) MISSA 2.0: an updated synthetic biology toolbox for assembly of orthogonal CRISPR/Cas systems. Sci Rep 7:41993 